\title{
Aprendizagem colaborativa mediada por tecnologia - um estudo de caso da plataforma aberta "ENEM na Rede"
}

\author{
Marcos Arrais e Silva' ${ }^{1}$, Jonice Sampaio de Oliveira ${ }^{1}$ \\ 'PPGI - Universidade Federal do Rio de Janeiro (UFRJ) \\ Rio de Janeiro - RJ - Brazil \\ \{marcos.arrais,jonice\}@gmail.com
}

\begin{abstract}
This paper presents the case study of the platform ENEM na Rede. An online, social and free tool for teaching and learning the curriculum content of the Brazilian high school exam (Exame Nacional do Ensino Médio ENEM). The artifact utilizes information visualization techniques, gamification, web data mining, and an intelligent tutoring system to build with the student an individualized syllabus with growth metrics and goals. The study was evaluated in a state school with a group of 98 students, from March to May 2019. The results show promising learning rates.
\end{abstract}

Resumo. Esse trabalho apresenta o estudo de caso da plataforma ENEM na Rede. Uma ferramenta online, social e livre para ensino e aprendizagem dos conteúdos curriculares do Exame Nacional do Ensino Médio - ENEM. O artefato utiliza técnicas de visualização de informações, gamificação, mineração de dados na web e um sistema de tutor inteligente para construir junto com o aluno um plano de estudos individualizado com métricas e metas de crescimento. $O$ estudo foi avaliado em uma escola estadual com um grupo de 98 estudantes, entre o período de março a maio de 2019. Os resultados encontrados demonstram índices promissores de aprendizagem.

\section{Cenário e Contexto}

Existem inúmeras iniciativas livres na internet como Khan Academy (pt.khanacademy.org), Coursera (www.coursera.org) e edX (www.edx.org), que apoiam o ensino formal com técnicas de autoaprendizagem, sejam através de plataformas de simulados, videoaulas ou mesmo discussões em um grupo aberto de uma rede social online. A grande dificuldade dessas plataformas é que não existe um método avaliativo que possa aferir o crescimento, erro, acerto e carência de parte do conteúdo programático. Muitas vezes, quando a aluno inicia um processo de estudo informal, ele não sabe por onde começar e salta unidades formativas que tem pré-requisito.

Nesse contexto, este estudo objetiva a construção de uma ferramenta (figura 1) que incorpore um método de autoaprendizagem, focado na preparação de estudantes para o Exame Nacional do Ensino Médio (ENEM). O artefato utiliza dados públicos da rede social on-line Facebook para classificar os conteúdos a serem estudados com base nas proficiências e deficiências do aluno. Para apresentar conteúdos de estudo ao aluno o sistema coleta websites e vídeos públicos nas plataformas Google e Youtube que possuam correlação com as deficiências de conteúdo apresentadas pelo aluno. Esse processo de mineração de dados da web é mediado por um sistema de classificação e filtro de informações, que é apresentado adiante. Ainda para garantir o envolvimento do aluno na plataforma, o sistema possui uma camada de gamificação, em que é necessário 
VIII Congresso Brasileiro de Informática na Educação (CBIE 2019)

Anais dos Workshops do VIII Congresso Brasileiro de Informática na Educação (WCBIE 2019)

realizar interações sociais, conquistar medalhas e realizar testes (quiz) com outros estudantes.

O ambiente foi validado com 98 estudantes da escola estadual Ordem e Progresso na cidade de Belo Horizonte -MG.

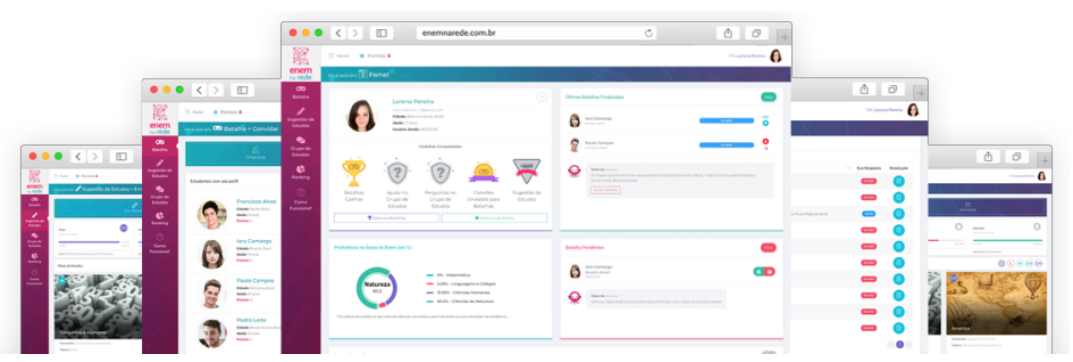

Figura 1: Interface gráfica da plataforma ENEM na Rede

\section{Desenvolvimento da Ferramenta}

Para garantir significativos ganhos de aprendizagem dos conteúdos das quatro grandes áreas do ENEM (linguagens e códigos, matemática, ciências humanas e da natureza), foi elencada uma estrutura de funcionamento da ferramenta que está definida em: i) gerar testes com questões no padrão ENEM; ii) avaliar os erros/acertos dos testes e definir proficiências e deficiências de conteúdos; iii) montar um plano de estudos individualizado com as necessidades de conteúdos do aluno; iv) oferecer conteúdos de repositórios externos para que o aluno utilize como apoio no processo de estudo; v) reiniciar esse processo de forma continua.

A primeira fase do desenvolvimento foi à mineração de questões de domínio público do ENEM de anos anteriores (2008 A 2018) e de universidades de todo o Brasil para compor a base de perguntas que seriam utilizadas para validar os conhecimentos do aluno. Essa foi uma das etapas mais importantes porque todo conteúdo adicionado ao banco de dados necessitava de uma adequação para encaixar com a matriz de competências do ENEM (INEP, 2015). Ao todo foram mapeadas 32 mil questões. Com a interface gráfica, finalizada e regras de negócio implementadas, deu-se início a construção dos robôs autônomos que funcionam como serviços da ferramenta. Tais agentes autônomos são responsáveis por coletar os conteúdos utilizados na plataforma e realizar rotinas autônomas em intervalos de tempo definidos, como por exemplo, calcular as proficiências e deficiências de alunos, além de extrair dados relevantes da rede social do aluno.

\subsection{Funcionalidades da Ferramenta}

O funcionamento da ferramenta está dividido em quatro requisitos. São eles:

- Batalha: principal área da plataforma. Aqui são apresentadas as questões no formato do ENEM e a partir dos erros e acertos dos alunos, o sistema calcula as proficiências e deficiências para construir um plano de estudo para o aluno. Essa área da plataforma é executada em duplas, onde um estudante convida outro para responder uma rodada de perguntas. Cada rodada é composta por 10 perguntas que fazem parte do escopo de deficiências do desafiador e do oponente; 
VIII Congresso Brasileiro de Informática na Educação (CBIE 2019)

Anais dos Workshops do VIII Congresso Brasileiro de Informática na Educação (WCBIE 2019)

- Sugestão de Estudos: é o campo onde são apresentados os planos de estudos do aluno. O sistema seleciona alguns assuntos relacionados às deficiências identificadas durante as batalhas. Após a revisão desses assuntos, o conteúdo é inserido novamente como batalha para que seja avaliado se houve o aprendizado;

- Grupo de Estudos: coleta as interações sociais dos estudantes em um grupo público de Facebook e analisa seu conteúdo. Caso os assuntos debatidos sejam pertinentes ao ENEM, a plataforma atribui pontos ao estudante no sistema de gamificação;

- Ranking: é onde o estudante pode ver seus pontos e sua colocação no sistema. Todas as atividades da ferramenta são pontuadas. Essa mecânica é responsável pelo maior envolvimento do aluno uma vez que suas conquistas são compartilhadas com outros usuários da rede.

\subsection{Arquitetura e Tecnologias da Plataforma}

A figura 3 demonstra a arquitetura conceitual da plataforma e mostra como os serviços e abordagens web estão interconectados para a construção de um artefato único. É importante observar que toda a interação do aluno acontece em um front-end web que usa os dados de usuário fornecidos pela plataforma Facebook.
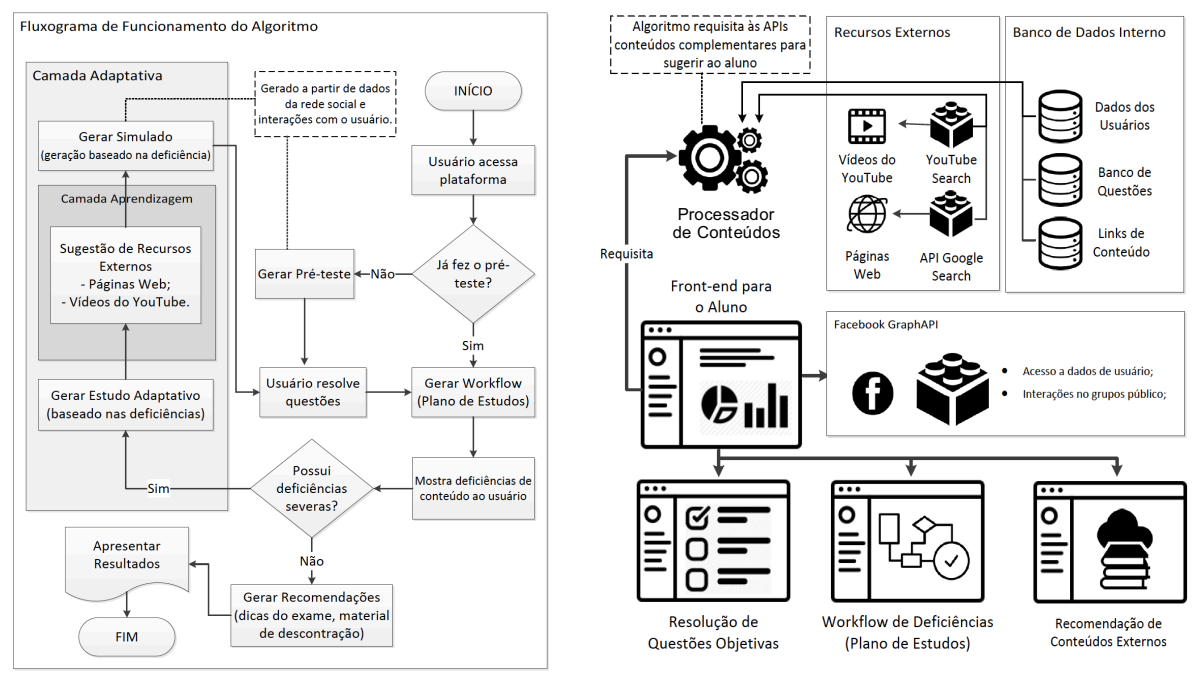

Figura 2: Proposta de Arquitetura Conceitual da Plataforma

Para o front-end do usuário foi criado um website responsivo que utilizou as tecnologias:

- Framework de programação: PHP/Lavarel;

- Motor de recuperação da informação: Elastic Search;

- Robôs autônomos: Python;

- Servidor: Apache $2+$ Linux Ubuntu;

- APIs: Facebook Graph API 3.0, Google Custom Search API e Youtube Search API. 
VIII Congresso Brasileiro de Informática na Educação (CBIE 2019)

Anais dos Workshops do VIII Congresso Brasileiro de Informática na Educação (WCBIE 2019)

\subsection{Processador de Conteúdos}

É responsável por percorrer todo o banco de questões e para cada questão minerar através da Google Search API e o do Youtube Search API conteúdos que atendem a critérios pré-definidos. O robô escolhe os conteúdos que irão compor a base referenciado na reputação atribuída pelas search engines. No caso da Youtube Search também são considerados as curtidas e inscrições que o vídeo e canal possuem. É importante ressaltar que somente conteúdos públicos da internet são indexados pelos robôs.

A abordagem para coleta de websites aproveita a inteligência do algoritmo de pagerank do Google Search. Sendo assim, o robô utilizando a API pública Google Search API extrai os resultados e classifica quais irão para a base. $\mathrm{O}$ termo de consulta é obtido através da extração das palavras-chave da questão combinadas com o assunto, o tópico e o subtópico da questão. A extração da palavra-chave utiliza o seguinte procedimento: $1^{\circ}$ - remoção de stop words; $2^{\circ}$ - stemming e lowercase; $3^{\circ}$ - contagem da frequência do termo; $4^{\circ}$ - seleção dos três termos mais frequentes. Para consolidar a query de busca são utilizados operadores booleanos e aspas para enclausurar expressões. Seguindo a estrutura: "assunto" $A N D$ "tópico" $A N D$ "subtopico" $A N D$ ("palavra-chave 1" OR "palavra-chave 2" $O R$ "palavra-chave 3").

Durante o processo de extração de dados via API o robô executa também o processo de classificação dos itens. Para que os endereços de website entregues pela Google Search API sejam selecionados, eles devem atender os seguintes critérios:

- O conteúdo deve ter mais de um ano que figura nos resultados de busca (obtido pelo tempo que o resultado figura nos resultados da Google Search);

- Deve estar em português;

- Obrigatório conter o assunto e tópico no corpo do texto.

Para os vídeos selecionados da plataforma Youtube:

- $\quad$ O saldo de curtidas (likes) deve ser maior que o de não-curtidas (unlikes);

- Devem existir comentários no vídeo;

- Deve ter um canal com outros vídeos associados.

É importante ressaltar que em ambas as consultas é respeitado o ranking que o sistema de busca classificou. Sempre que o número mínimo de resultados $(2$ websites e 2 vídeos) não é atingido, o robô refaz a busca removendo primeiramente o subtópico, e depois o tópico. Se depois de remover subtópico e tópico as quantidades mínimas não forem cumpridas, o algoritmo cadastra a quantidade encontrada e envia um e-mail para o administrador do sistema com a query de busca e o código da questão para que o processo seja realizado manualmente.

\subsection{Processador de Proficiências e Deficiências}

Esse robô é responsável por processar os conteúdos que o aluno tem deficiências e sugerir um plano de estudos a ele. Sua instância é lançada sempre que um aluno realiza um teste dentro da plataforma. A parte fundamental do processo está em somar ao final o grau de dificuldade do item $\left(D_{i}\right)$ estabelecido por Pasquali (2003) como: 


$$
D_{i}=\frac{C_{i}-\frac{E_{i}}{K_{i}-1}}{N_{i}}
$$

\section{Equação 1 - Nível de dificuldade com influência da escolha aleatória por Pasquali (2003)}

Onde a dificuldade de um item $\left(D_{i}\right)$ é representada pelo número de indivíduos que responderam corretamente um item $\left(C_{i}\right)$ menos a razão entre o número de alunos que erraram o item $\left(E_{i}\right)$ pelo número de respostas do item $\left(K_{i}\right)$ menos um, dividido pelo número de indivíduos que realizaram o teste $\left(N_{i}\right)$.

Baseado nos erros/acertos do teste, o robô utiliza o algoritmo de proficiência/deficiência para aferir quais conteúdos demandam atenção.

Quando um aluno acerta uma questão, é necessário invocar os graus de proficiência históricos para aquele tópico e subtópico de conteúdo (Valores de Proficiência - $V_{p}$, no qual $\boldsymbol{n}$ é o total de registros da base, para aquele tópico e subtópico), que estão gravados na base de dados.

Sendo assim para a equação de proficiência (Arrais, 2019) temos:

$$
\text { Proficiência }=\log \left(\frac{\sum_{i=0}^{n} V_{p}+1}{\sum_{i=0}^{n} V_{d}+1}\right)+D_{i}
$$

\section{Equação 2 - Cálculo de proficiência em um tópico específico de conteúdo}

Na equação de deficiência (Arrais, 2019), o grau de dificuldade da questão tem um peso maior do que na proficiência. Essa adequação faz com que o estudante tenha que acertar mais de uma questão para que seu grau seja convertido de deficiência em proficiência.

$$
\text { Deficiência }=\log \left(\frac{\sum_{i=0}^{n} V_{d}+1}{\sum_{i=0}^{n} V_{p}+1}\right)+1+D_{i}
$$

\section{Equação 3 - Cálculo de deficiência em um tópico específico de conteúdo}

Quando uma questão não possui a dificuldade $\left(D_{i}\right)$ calculada, por ainda não ter uma base de respondentes de 100 usuários o coeficiente de dificuldade é alterado para o valor 1 de deficiência e 0.5 quando se tratar de proficiência. $\mathrm{O}$ coeficiente de dificuldade $\left(D_{i}\right)$ é recalculado sempre que um teste é processado, mantendo o equilíbrio da base, visto os novos usuários e testes que são realizados durante a execução do artefato.

Os valores de proficiência e deficiência são utilizados para definir quais conteúdos devem ser apresentados no workflow. Quanto maior a deficiência, maior a prioridade no ranking de conteúdos. Quando o saldo de proficiência é maior que o de deficiência o conteúdo é transferido para uma área de itens revisados.

\subsection{Processador de Interações Sociais}

Responsável por quantificar a participação do aluno no grupo de estudos da plataforma. O grupo de estudos opera dentro da rede social on-line (RSO) Facebook e, diariamente, 
VIII Congresso Brasileiro de Informática na Educação (CBIE 2019)

Anais dos Workshops do VIII Congresso Brasileiro de Informática na Educação (WCBIE 2019)

a cada 2 horas, o robô visita a rede, extrai as participações do aluno e verifica se elas têm correlação com algum conteúdo da base. Caso positivo o aluno é bonificado com uma quantidade de pontos no sistema de gamificação da plataforma.

Para processar as interações dos alunos no grupo, diariamente a cada duas horas um robô conecta-se a Graph API do Facebook e lê todas as postagens realizadas no grupo. As postagens são divididas em dois grupos: perguntas e respostas. As perguntas são postagens realizadas no fluxo principal do grupo, conhecido como feed. As respostas são comentários adicionados a perguntas do feed. Para cada post realizado, o algoritmo verifica qual é o autor do conteúdo e processa o texto para identificar se está relacionado a algum conteúdo da matriz de referência do ENEM. Caso positivo, ele salva o conteúdo na área de "Grupo de Estudos" do aluno e atribui pontos pela publicação.

O processamento do conteúdo é realizado por técnicas de recuperação da informação, que extrai do texto do aluno as palavras-chave e compara com a base de dados de questões do artefato. A primeira fase de construção do algoritmo foi a indexação de todas as palavras-chave das questões, agrupadas por subtópico e tópico. Sempre que um novo conteúdo é recuperado, suas palavras-chave são comparadas às palavras-chave da base indexada. Esse processo retorna um coeficiente de correlação: Quanto mais alto, maior a probabilidade de os conteúdos serem relacionados. Para a postagem do aluno, os tratamentos realizados nas strings foram: $1^{\circ}$ - remoção de stop words; $2^{\circ}$ - stemming e lowercase; $3^{\circ}$ - contagem da frequência do termo. Já para o processo de indexação, que é usado como a base de comparação para a primeira fase, foram considerados os seguintes passos:

- Junção de todas as palavras-chave das questões por tópico;

- Remoção das strings duplicadas para o atributo palavras-chave, stop words, stemming e lowercase;

- Junção de todos os subtópicos das questões por tópico;

- Remoção das strings duplicadas para o atributo subtópico, stop words, stemming e lowercase;

- Agrupamento por tópico e assunto;

- Indexação dos termos utilizando o método Term frequency-inverse document frequency selection (TF-IDF) (Leginus, 2015);

Após o processo de indexação, o robô compara se o texto digitado pelo aluno tem correlação com os textos indexados na base, retornando um coeficiente.

Percebeu-se que somente aplicar o método TF-IDF padrão não geraria uma correlação semântica. Foi então que o algoritmo foi adaptado para funcionar com pesos individuais em cada atributo (tópico, subtópico e palavra-chave). Além de acrescentar o peso individual foi adicionado um fator de normalização do TF que utiliza o tamanho do documento como um critério de importância. Essa normalização garantiu melhorias significativas da correlação. Enquanto alguns documentos indexados possuem poucas palavras-chave, visto o baixo número de questões relacionadas na base, outros documentos possuem muitas questões relacionadas, resultando em um grande número de palavras-chave e consequentemente no aumento do tamanho do documento. $\mathrm{O}$ 
VIII Congresso Brasileiro de Informática na Educação (CBIE 2019)

Anais dos Workshops do VIII Congresso Brasileiro de Informática na Educação (WCBIE 2019)

processo de normalização permitiu que os documentos com tamanhos diferentes pudessem ser comparados. A equação final foi definida matem:

$$
\begin{gathered}
T F-I D F \text { Normalizado }=T F * I D F \\
T F=\sqrt{T F_{x, y}} * \frac{1}{\sqrt{l}} * w \\
I D F=\left(\log \frac{N+1}{d f+1}+1\right) * w
\end{gathered}
$$

\section{Equação 4 - Adaptação do TD-IDF para uso de pesos variáveis e normalização}

Onde $T F_{x, y}$ é a frequência de uma palavra-chave $X$ em um documento $Y, N$ é o total de documentos na base, e $d f$ é o número de documentos que contém a palavra-chave $X$, o $w$ é o peso que será variável por atributo, trazendo a possibilidade de definir uma maior importância, por exemplo ao subtópico. O $l$ é o tamanho do documento em palavras.

Os documentos são indexados e seus atributos receberam os seguintes fatores de importância: topico $_{w}=6 ;$ subtopico $_{w}=4 ;$ palavras - chaves $_{w}=0.6$. Esse coeficiente foi ajustado realizando vários testes para processar a eficiência. É possível observar que se uma string está presente no tópico do documento, ela ganha um alto fator de importância, o que faz sua nota de correlação crescer/aumentar.

\subsection{Tutoria do aluno mediado por agente}

Durante a construção das interfaces observou-se que o artefato precisava dialogar constantemente com o usuário, informando o término de atividades, instruindo sobre qual conteúdo ele deveria visitar, quais conteúdos foram importados no "Grupo de Estudos". Esse diálogo contínuo trouxe a necessidade de criar uma representação visual que mediasse à comunicação do artefato com o aluno. Assim surgiu o tutor da plataforma ENEM na Rede, que recebeu o nome de "Aê", que em tupi-guarani significa "Amigo". Como o funcionamento do artefato já era baseado na operação de três serviços automatizados, os robôs de conteúdo, proficiência/deficiência e interações sociais, o tutor recebeu a identidade de um robô, com traços simples e utilizando as cores do artefato. A figura 3 ilustra algumas situações de diálogo entre o "Aê" e o aluno.

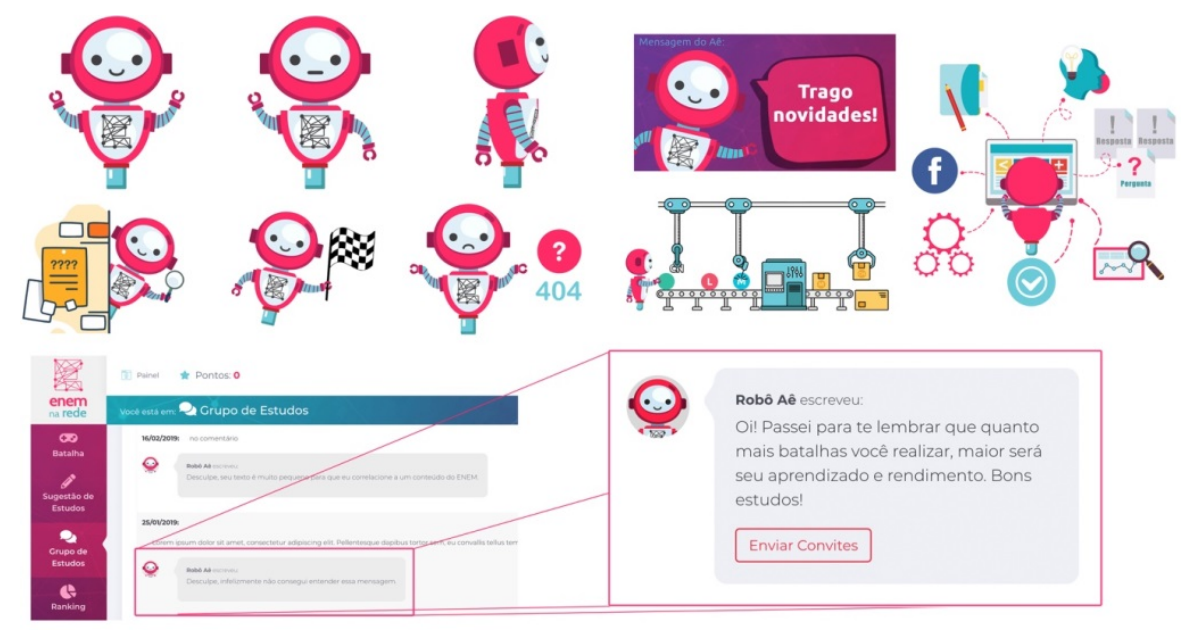

Figura 3 - Ilustrações do mascote "Aê" na plataforma ENEM na Rede 
VIII Congresso Brasileiro de Informática na Educação (CBIE 2019)

Anais dos Workshops do VIII Congresso Brasileiro de Informática na Educação (WCBIE 2019)

\subsection{Gamificação}

Foi observado que o processo cíclico e contínuo das atividades do artefato poderia não gerar muito engajamento e participação por parte dos alunos, desmotivando o uso do artefato, uma vez que o processo de operação ficava restrito a: responder questão; acessar conteúdos; repetir o processo. Observou-se então a necessidade de uma técnica mais envolvente para o uso do sistema, a assim as dinâmicas de gamificação foram incorporadas ao projeto, gerando duas mecânicas sociais para envolver o aluno no uso do artefato, são elas: testes baseados em batalhas entre estudantes e um sistema de pontos com medalhas (badges) para premiação baseado em participação.

Tabela 1. Medalhas (Badges) que podem ser conquistados na plataforma

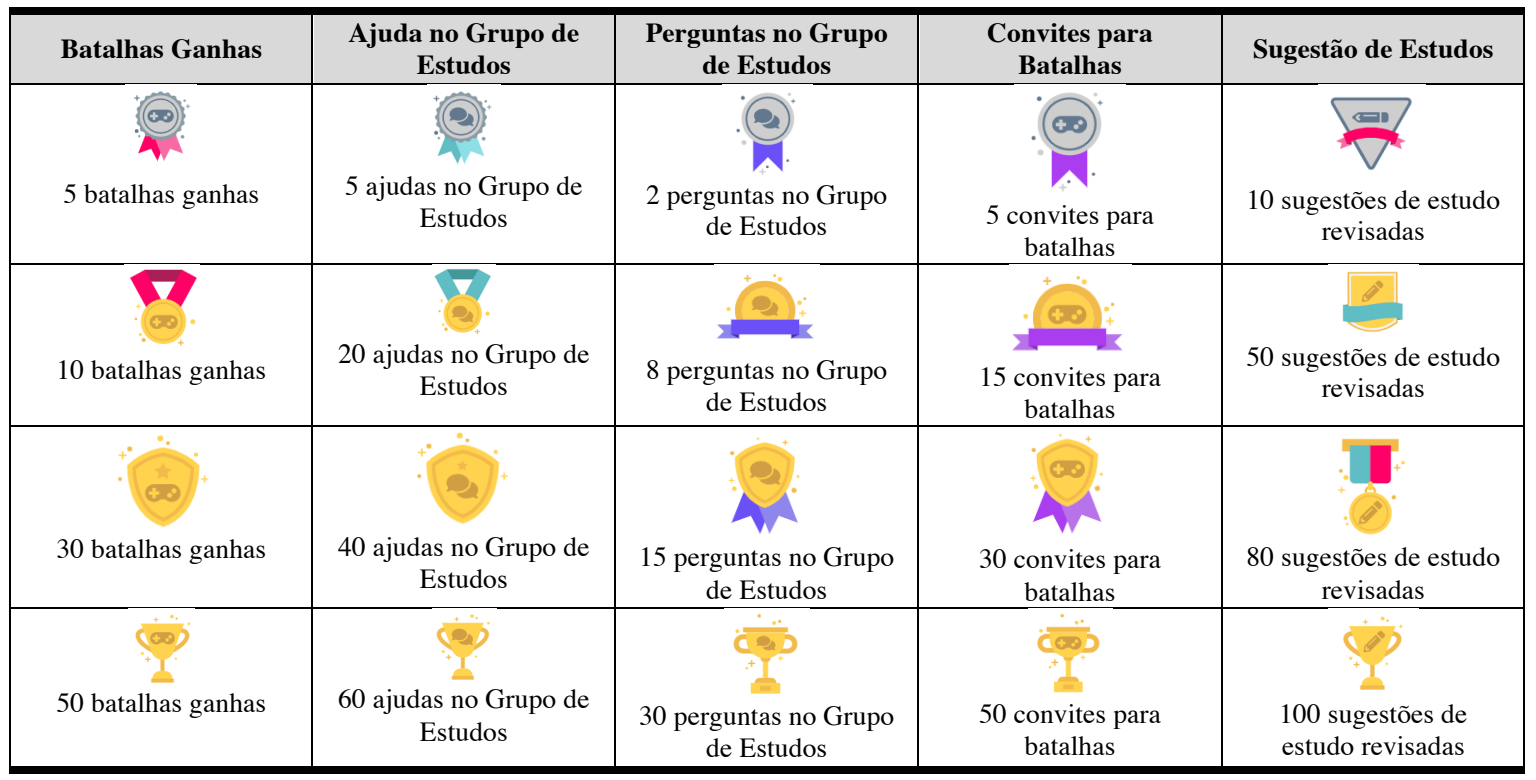

\section{Resultados e Conclusão}

Durante o período de três meses (março a maio de 2019) 43 estudantes testaram a ferramenta e foram avaliados em simulados presenciais, realizados em duas etapas. $\mathrm{O}$ primeiro simulado, aplicado antes do ingresso dos alunos na plataforma foi utilizado para aferir os conhecimentos prévios dos alunos. Um grupo de controle de 55 alunos, que não utilizou a plataforma, também realizou o simulado para que seus dados pudessem ser confrontados com o dos alunos que ingressaram na plataforma. Ao final do terceiro mês foi aplicado outro simulado no mesmo modelo do primeiro. O grupo que utilizou a plataforma apresentou ganhos de aprendizagem sobre o segundo grupo entre $2,5 \%$ a 3\% dependendo da área de conhecimento avaliada. 
VIII Congresso Brasileiro de Informática na Educação (CBIE 2019)

Anais dos Workshops do VIII Congresso Brasileiro de Informática na Educação (WCBIE 2019)

\section{Demonstração da Ferramenta}

Foi desenvolvido um vídeo tutorial da ferramenta que apresenta todas as áreas de sistema. Esse vídeo pode ser acessado pelo link: https://youtu.be/4pyhTwEFjMY

\section{Referencias}

ARRAIS, Marcos. Processamento de conteúdo, proficiências, deficiências e de interações sociais para a preparação para o Exame Nacional do Ensino Médio. Tese (Doutorado em Informática). Programa de Pós-graduação em Informática, Universidade Federal do Rio de Janeiro. Rio de Janeiro, 2019.

INEP. Matriz de Referencia do ENEM. 2015. Disponível em: $<$ http://download.inep.gov.br/download/enem/matriz_referencia.pdf> Acesso em: 20/10/2019

LEGINUS, Martin; ZHAI ChengXiang; DOLOG, Peter. Personalized generation of word clouds from tweets. J. Assoc. Inf. Sci. Technol., p. n/a-n/a, 2015.

MORAES, André. BELLUZZO, Walter. O diferencial de desempenho escolar entre escolas públicas e privadas no Brasil. Nova Economia. vol.24 no.2 Belo Horizonte Mai/Ago. 2014.

PASQUALI, Luis. Psicometria: teoria dos testes na psicologia e na educação. Petrópolis: Vozes, 2003. 397 p. 